\title{
The Emerging Role of Carbon-Ion Radiotherapy
}

\author{
Daniel K. Ebner and Tadashi Kamada* \\ Research Center for Charged Particle Therapy, National Institute of Radiological Sciences, Chiba, Japan
}

Carbon-ion radiotherapy (CIRT) has progressed rapidly in technological delivery, indications, and efficacy. Owing to a focused dose distribution in addition to high linear energy transfer and subsequently high relative biological effect, CIRT is uniquely able to target otherwise untreatable hypoxic and radioresistant disease while opening the door for substantially hypofractionated treatment of normal and radiosensitive disease. CIRT has increasingly garnered international attention and is nearing the tipping point for international adoption.

Keywords: particle beam therapy, carbon-ion radiotherapy, adaptation, performance, radioresistance

\section{INTRODUCTION}

OPEN ACCESS

Edited by:

Marco Durante,

GSI, Germany

Reviewed by:

William F. Hartsell,

Northwestern Medicine Chicago

Proton Center, USA

Takashi Nakano,

Gunma University, Japan

*Correspondence:

Tadashi Kamada

kamada.tadashi@qst.go.jp

Specialty section:

This article was submitted to Radiation Oncology,

a section of the journal

Frontiers in Oncology

Received: 25 January 2016

Accepted: 23 May 2016

Published: 07 June 2016

Citation:

Ebner DK and Kamada T (2016) The

Emerging Role of Carbon-lon

Radiotherapy.

Front. Oncol. 6:140.

doi: 10.3389/fonc.2016.00140
In 1952, the first human patients were treated by John Lawrence and Cornelius Tobias with helium and deuteron particle beams (1). Subsequently, interest in particle beams expanded, with proton facilities emerging throughout the world. However, as the biological impact of protons mirrored that of X-ray therapy, attention turned to heavier ions due to a higher biological impact owing to higher linear energy transfer (LET) (2). In 1975, with the installation of the BEVALAC to the Lawrence Berkeley Laboratory (LBL), extensive research into the clinical potential of heavy-ion beams more formally began (3).

In response to the initial successes at $\mathrm{LBL}$, in 1984, the Japanese government began construction on the world's first heavy-ion facility designated for medical use at the National Institute of Radiological Sciences (NIRS), staffing it with scientists returning from the BEVALAC and LBL. The Heavy Ion Medical Accelerator in Chiba (HIMAC) was completed in 1993, with clinical trials in carbon-ion radiotherapy (CIRT) beginning in June 1994.

Similar to the BEVALAC, the HIMAC provided for passive-beam irradiation. NIRS was alone in offering CIRT until 1997, when the GSI Carbon-Ion Radiotherapy Facility in Germany came into operation, pioneering raster scanning heavy-ion beams in clinical practice. GSI treated 440 patients with good results before its closure in 2008 (4). NIRS completed development of a pencil-beam raster scanning (PBS) treatment facility in 2012, and initial clinical trials are promising.

Developments in diagnostic technologies have enabled new therapeutic applications, such as markerless respiration-gated PBS irradiation. The enhanced radiobiological effect of the carbonion, concentrated and converged into a highly conformal dose distribution coinciding with targetrespiratory movement, has allowed for medical care of radioresistant, previously untreatable disease (5-7). Further, these advantages have provided for hypofractionated radiotherapy of more common diseases, as well as improved adverse effect profiles, in comparison to conventional therapy. Altogether, this has lead to excellent treatment results in numerous diseases.

To date, nearly 70 protocols have been conducted at NIRS to delineate CIRT efficacy, safety, optimal treatment indications, and dose fractionation (8). Protocols begin with phase I dose-escalation studies focused on minimizing adverse effects. This is followed by phase II evaluation of treatment efficaciousness with longitudinal follow-up. If feasible, protocols exploring hypofractionation 
follow. Initial protocols began with low doses and an average of 18 fractions, but after critical review of technical and clinical data, today cases average 11-12 fractions. One or two total fractions are possible for indicated lung and liver disease, respectively. As such, the Hospital of the NIRS has reached a treatment capacity of between 900 and 1000 patients per year.

Carbon-ion radiotherapy facilities and faculty continue to grow in number and experience, with $8+$ operational centers and over 15,000 patients treated to date (9). In Japan, in addition to the four heavy-ion radiotherapy facilities in operation prior to 2015, the Kanagawa Cancer Center's carbon-ion facility began treatment in December 2015, and plans exist to construct facilities in Osaka City as well as Yamagata and Okinawa Prefectures. In light of the concentration of CIRT facilities in Japan, the Japan Carbon-ion Radiation Oncology Study Group (J-CROS) was organized to coordinate multi-institutional studies moving forward. Internationally, Austria will open a CIRT center in 2017, with centers in South Korea, Taiwan, China, and the United States in various states of development. Further, the clinical successor to the GSI Carbon-Ion Radiotherapy Facility, the Heidelberg Ion Therapy Center (HIT), has begun a number of randomized trials, testing carbon(-boost) versus other irradiation modalities (10-14).

In this paper, we aim to update on the expanding role of CIRT in cancer treatment as of 2016.

\section{FEATURES OF CIRT}

In comparison with conventional radiotherapy, particle beams possess different physical and biological characteristics that must be weighed when considering treatment. While conventional radiation generally passes continually through a biological target, with dose delivered roughly equivalently throughout the beam path, particle beams release energy at the inverse of their velocity (Figure 1). Particle beams thus deliver a lower entry dose, depositing the majority of their energy at the flight path terminus, yielding an asymptotic dose peak (the "Bragg Peak") (15). This allows for a dose concentration distribution impossible with conventional irradiation methods.

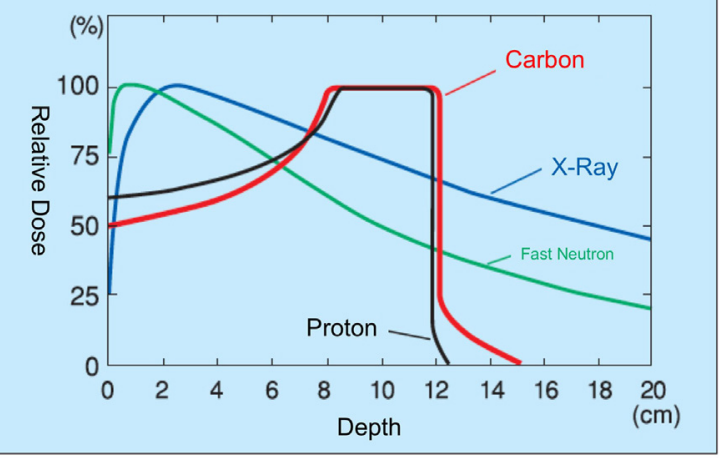

FIGURE 1 | Dose distribution of heavy-particle beams.
Today, proton dominates particle therapy. However, the larger mass of carbon results in decreased beam scattering, yielding a sharper dose distribution border with minimal penumbra (16). Radiobiologically, carbon-ion beams result in two to three times the relative biological effect (RBE; the biological effectiveness of one type of ionized radiation relative to another, given the same amount of absorbed energy) of proton and conventional irradiation methods (17). In comparison with photon therapy, CIRT does not show an oxygen effect, sublethal damage repair, and has less cell-cycle-related radiosensitivity.

These unique characteristics formed the rationale in initially applying carbon to radioresistant and/or hypoxic disease. Further indications then arose: the sharp dose distribution allows therapeutic dose delivery to disease juxtaposed with vital, radiosensitive organs (18-20). With radionormal or radiosensitive disease, short-term hypofractionated treatment becomes possible, owing to diminished dose delivered to healthy tissue.

\section{CARBON-ION RADIOTHERAPY TREATMENT}

To date, over 9000 patients have undergone CIRT at NIRS, with 12,000 across all facilities in Japan and over 15,000 worldwide. In 2003, upon review of the first 9 years of NIRS' clinical trials, the Japanese government allowed CIRT availability to the general public. CIRT has demonstrated efficacy against prostate, head and neck, lung, and liver cancers, bone and soft tissue sarcomas, locally recurrent rectal cancer, and pancreatic cancer, including locally advanced disease $(8,19,21)$. Below, we provide a brief summary of the current most common indications and the data supporting their treatment.

At NIRS, over 2000 prostate cancer patients have been treated with CIRT, comprising approximately a fourth of CIRT-treated cases. Half of these cases are considered high risk at the time of treatment (determined by high PSA, T3 status, or high Gleason score). Initially, dose escalation in 20 fractions was performed, followed by investigation of hypofractionation. From 2007 to 2013, 781 patients were treated with 57.6 Gy (RBE) delivered in 16 fractions, with 5-year overall survival (OS) and biochemical relapse-free rates of 96.9 and $92.8 \%$, respectively. No grade 3 or higher toxicity was seen. In 2014, treatment shifted to 12 fractions [51.2 Gy (RBE)] delivered over 3 weeks, yielding 100\% causespecific survival at a median follow-up of 32.3 months. At this dose-fractionation, no grade 3 or greater acute or late toxicities were observed, comparing favorably to conventional radiotherapy. Long-term data are pending, and further hypofractionation is being considered (22-24). Internationally, two randomized trials comparing proton and carbon are under recruitment at HIT (10).

Highly radioresistant non-squamous-cell carcinomas accounted for the majority of head and neck disease treated at NIRS, consisting of $11 \%$ of CIRT cases there. In a review of 240 patients (243 lesions), over a 9-year period, excellent results have been reported. $91 \%$ of patients received $57.6 \mathrm{~Gy}(\mathrm{RBE})$ with the remainder receiving 64.0 Gy (RBE), both delivered in 16 fractions. Approximately half of the high-dose group consisted of bone and soft tissue sarcomas of the head and neck. The 5-year 
local control (LC) rate was $68 \%$ across all head and neck cancers, with OS of $47 \%$ (LC/OS histological breakdown: 75/35\% mucosal malignant melanoma, 73/68\% adenoid cystic carcinoma (ACC), $73 / 56 \%$ adenocarcinoma, $24 / 36 \%$ sarcomas, $61 / 31 \%$ papillary adenocarcinoma, and $61 / 17 \%$ squamous cell carcinoma). Acute grade 3 skin and mucosal reactions were seen in 15 (6\%) and 24 (10\%) of patients, respectively, with no acute grade 4 or higher toxicity seen. No late skin grade 3 or greater toxicities were noted. Late mucosal side effects included no grade 3, but four cases of grade 4 ipsilateral blindness $(25,26)$. In 109 head-and-neck-based malignant mucoscal melanoma patients treated concomitantly with dacarbazine, nimustine, and vincristine (DAV), a 5-year LC rate of $82 \%$ with OS of $52 \%$ was achieved versus $33 \%$ OS with carbon alone (27). At HIT, carbon ions were used as boost in ACC, achieving $78 \% \mathrm{LC}$ at 4 years, with rates of severe late toxicity $<5 \%$ (28).

A majority of bone and soft tissue tumors are radioresistant and form a prototypical disease for CIRT treatment. Thus, despite being comparatively rare, these make up $11 \%$ of CIRT cases at NIRS. In particular, in both the skull base and trunk, chordoma, osteosarcoma, spinal tumors, and retroperitoneal tumors treated with CIRT have demonstrated satisfactory results (27, 29-33). Skull base and paracervical disease treated with 48.0-60.8 Gy (RBE) in 16 fractions yielded an overall LC and OS rate of 86 and $85 \%$, respectively (LC/OS: $87 / 90 \%$ chordomas, $81 / 76 \%$ chondrosarcomas, $89 / 73 \%$ olfactory neuroblastomas, and $83 / 86 \%$ meningiomas). $24.5 \%$ of patients experienced grade 2 or greater radiation-induced brain injury (RIBI) $(7.0 \%$ symptomatic), with a single case of grade 4 RIBI $(27,34,35)$. This reinforced similar results from GSI, where LC of $70 \%$ at 5 years in chordoma and $87 \%$ at 4 years in chondrosarcomas, with limited toxicity, were achieved $(36,37)$. Randomized trials at HIT for these diseases are underway. In unresectable primary spinal sarcoma, following a dose of 52.8-70.4 Gy (RBE) in 16 fractions, 5-year LC and OS were 79 and 52\%, respectively, with smaller disease $\left(<100 \mathrm{~cm}^{3}\right)$ demonstrating 100\% LC. Three patients $(6 \%)$ experienced a grade 3 or greater adverse effect, and seven experienced vertebral body compression (32). In unresectable retroperitoneal sarcoma, following dosing of 52.8-73.6 Gy (RBE) in 16 fractions, 5-year LC and OS was 69 and $50 \%$, respectively. No grade 3 or greater toxicity was noted (33). In unresectable truncal osteosarcoma, following a median 70.4 Gy (RBE) applied in 16 fractions, LC of $62 \%$ and OS of $33 \%$ was seen, with no grade 3 or greater toxicity noted. Worse outcomes were seen in patients with a clinical target volume $>500 \mathrm{~cm}^{3}$ (31). At HIT, locally unresectable osteosarcomas are treated with carbon and chemotherapy in an ongoing trial that includes the only cohort of CIRT-treated pediatric patients. Results are forthcoming $(11,12)$.

With lung and liver cancers, the improved dose distribution and strong RBE of CIRT led to prospective trials in hypofractionation, yielding excellent results (20, 38-40). Lung cancers encompass $11 \%$ of cases at NIRS, and currently, single-fraction delivery of $50 \mathrm{~Gy}$ (RBE) is indicated for Stage I, T1 and T2 non-small-cell disease. This has demonstrated a 5-year LC rate of $80.4 \%$ for patients receiving doses of 36.0 Gy (RBE) or more (T1: $86.0 \%$ and T2: $71.7 \%$ ), with 5 -year OS of $56.3 \%$. For patients receiving 48 or 50 Gy (RBE), 2-year LC and OS were both $95 \%$ (39). The first non-Japanese lung cancer CIRT trial, at HIT, will be a prospective clinical trial on patients with chest wall infiltration (10). Hepatocellular carcinoma, making up 10\% of CIRT indications, leads to notably poor survival rates due to inherent radiosensitivity of the liver combined with poor resectability (41). Current hypofractionation efforts led to a two-fraction regime consisting of $45.0 \mathrm{~Gy}$ (RBE). This has yielded OS and LC rates of 71 and $83 \%$ at 3 years, respectively. No grade $3+$ reactions were noted in a $45 \mathrm{~Gy}$ (RBE) or higher dose treatment group (42). Of note, four-fraction, 52.8-Gy (RBE) treatment of tumors lying near to the porta hepatis has yielded good control: 5-year LC of $87.8 \%$ with OS of $22.2 \%$ without similar side-effect profiles to non-porta hepatis cases (20). The PROMETHEUS-1 trial at HIT reported initial results in 2013: at 11 months, LC of 100\% was achieved with no severe adverse events reported (43).

Locally recurrent rectal cancer (5\% of cases), pancreatic cancer ( $4 \%$ of cases), and cervical adenocarcinoma and related cancers (gynecological tumors encompass 3\% of cases) all demonstrate degrees of radioresistance, but CIRT has demonstrated excellent performance in treating these diseases. A phase I/II dose-escalation study of 170 recurrent rectal cancer patients was performed at NIRS, with escalating dose between 67.2 and 73.6 Gy (RBE) delivered over 16 fractions in 4 weeks. LC at 3 years was $92 \%$ for 73.6 Gy (RBE), with OS of $59 \%$ at $73.6 \mathrm{~Gy}(\mathrm{RBE})$ at 5 years. No acute grade 3 or greater adverse events were seen, with two grade 3 late skin and one grade 3 late gastrointestinal reaction noted $(44,45)$. The forthcoming PANDORA-01 trial at HIT will further evaluate use of carbon in the setting of recurrent rectal cancer (13). The results for locally advanced pancreatic cancer have drawn international attention with combined CIRT-gemcitabine therapy, yielding a 1- and 2 -year freedom from local progression rate (FFLP), evaluated by ${ }^{18} \mathrm{FDG}$ uptake, of 63 and $30 \%$, with OS at 1 and 2 years of 73 and 35\%, respectively. When limited to Stage III disease, 2 -year FFLP and OS improved to 40 and $48 \%$, respectively. $53 \%$ of patients experienced grade 3-4 hematological toxicity, and $7 \%$ experienced grade 3 anorexia. One case (1\%) of grade 3 intratumoral infection was noted. None of these reactions were life-threatening (21). The forthcoming PHOENIX-01 trial at HIT will evaluate advanced pancreatic cancer treatment with scanning carbon-ion beam irradiation in combination with gemcitabine (14). With regard to cervical cancer, 58 locally advanced adenocarcinoma cases were treated in a doseescalation study [62.4-74.4 Gy (RBE) in 20 fractions] between 1998 and 2010, with 5 -year LC of $54.5 \%$ and OS of $38.1 \%$. One patient experienced a grade 4 rectal complication, with no other grade 3 or higher toxicities reported (46).

Radiotherapeutic treatment of brain malignancies remains a substantial challenge. Combs and colleagues conducted a pooled analysis of HIT and Japanese data regarding the usage of carbonion boost $(\mathrm{CIB})$ in the treatment of anaplastic astrocytoma (AA) and glioblastoma (GBM) (47-49). Postoperatively, 50-Gy photon with nimustine hydrochloride was administered, with 16.824.8 Gy (RBE) CIRT provided as boost. In GBM and AA, median OS was 18 and 35 months with CIRT versus 14 and 39 months with standard postoperative radiochemotherapy (RCT) with 
temozolomide. Progression-free survival of GBM and AA were 6 and 6 months (RCT) and 8 and 34 months (CIRT), respectively. The potential benefit of CIRT noted is under further evaluation in the CLEOPATRA trial at HIT (47).

Overall, CIRT has demonstrated good adaptability for difficult-to-treat, radioresistant disease, while allowing accelerated, hypofractionated treatment of other disease. Distant metastasis remains a challenge, but initial evaluations of CIRT concurrent with chemotherapy has demonstrated satisfactory performance $(21,27)$.

\section{FUTURE DIRECTIONS}

The future of charged particle therapy as of 2016 appears bright, with implementation of respiration-gated fast PBS (50), markerless tracking (51), a range-shifter-free multiple-energy modulation system, and completion of the second carbon-ion rotating gantry in the world at NIRS, following the first at Heidelberg. Nine plus new CIRT centers are opening worldwide. However, the high cost associated with the construction, maintenance, and operation of CIRT facilities, as well as the corresponding costs in staff development and support, presents a challenge for extension of the technology outside of the developed world.

As such, a great deal of work remains. Development in costsaving and improved miniaturization of existing technology is necessary. To date, these efforts have produced CIRT accelerator and synchrotrons at one-third the cost and size of the original HIMAC, which are in operation at the Saga-HIMAT, Gunma University, and Kanagawa Cancer Center i-ROCK facilities. Superconduction technology allowed for the recently completed rotating gantry at NIRS to be built with a length and diameter of 13 and $5.5 \mathrm{~m}$, respectively, versus the existing gantry at HIT, which is $25 \mathrm{~m} \times 13 \mathrm{~m}$ (52). Ongoing development aims to further employ superconducting technology in the accelerator and overall device, producing a total CIRT setup dubbed the "Super MINIMAC" that will fit within $20 \mathrm{~m}^{2}$. Meanwhile, limited research has been published on the cost-efficacy of CIRT (53-55), which would appear to improve with each new technological development; focused evaluation may be necessary to facilitate international development.

In Japan, CIRT is available as a private treatment to the general public. Discussion to extend national insurance coverage to CIRT is ongoing. However, despite the current cost burden for patients (3.2 million yen/ 28,000 USD for a treatment course, regardless of fractionation; in Germany, treatment costs $\sim 1000$ Euro per fraction), the number of patients from both within and outside Japan continues to increase.

\section{REFERENCES}

1. Tobias CA, Anger HO, Lawrence JH. Radiological use of high energy deuterons and alpha particles. Am J Roentgenol Radium Ther Nucl Med (1952) 67:1-27.

2. Curtis SB. Plans for the high-energy, heavy-ion facility (BEVALAC) at Berkeley. Eur J Cancer (1974) 10:388.

3. Castro JR, Quivey JM, Lyman JT, Chen G, Phillips TL, Tobias CA. Radiotherapy with heavy charged-particles at Lawrence Berkeley Laboratory. J Can Assoc Radiol (1980) 31:30-4
Clinically, as the majority of cases treated with CIRT in the world were treated at NIRS, the majority of available clinical data is focused at a single institution spread over 20 years (19). As center numbers increase, multi-institutional trials and randomized, internationally coordinated trials may begin.

An international ecosystem supporting and interweaving CIRT clinical, physical, and biological development is also necessary. It is known that the LET of particle beams are nonhomogenous throughout the irradiated region, yielding variations in RBE (56). As carbon is a high LET beam, these variations are more appreciable than with low LET proton irradiation. Due to a risk of consequent under- or over-treatment and toxicity, complex dosing models are required in the use of heavy ions. Of particular note, the RBE model varies between international institutions. Within Japan, the MKM2010 model, a revision of the Microdosimetric Kinetic Model, has been developed and implemented (57). In Europe, versions of the local effect model (LEM) are dominant. Efforts to improve international standardization are progressing, with work by Fossati and colleagues providing for MKM2010 dose translation to the LEM model and vice versa (58). Improved model accuracy and careful manipulation of the high LET/RBE regions may enable LET painting of tumors (59). This "intensity modulated carbon therapy" may further improve dose distribution, and research to this end is underway.

\section{CONCLUSION}

Since 1970, heavy-ion radiotherapy has progressed rapidly in technological delivery and, consequently, in indications and efficacy. The ability for the carbon-ion beam to offer short-term, minimally invasive, function-, tissue-, and form-sparing treatment has garnered international attention, with the technology nearing the tipping point for international adoption. Technically, enhanced international collaboration is needed to produce an intercenter translatable dosing model consensus and to enhance results at the common borders between radiobiology and particle physics. Societally, cost and access to treatment remains a challenge, particularly in developing countries. However, evidence continues to mount for the superiority of carbon in the treatment of radioresistant, hypoxic disease. Coupled with the opportunity for substantially abbreviated treatment of common disease, carbon-ion radiotherapy looks increasingly appealing as a treatment modality deserving worldwide availability.

\section{AUTHOR CONTRIBUTIONS}

\section{DKE and TK wrote and edited the manuscript.}

4. Kraft G. History of the Heavy Ion Therapy at GSI. (2013). p. 1-17. Available from: https://three.jsc.nasa.gov/articles/Krafts_GSI.pdf

5. Kanai T, Endo M, Minohara S, Miyahara N, Koyama-ito H, Tomura H, et al. Biophysical characteristics of HIMAC clinical irradiation system for heavyion radiation therapy. Radiat Oncol Biol (1999) 44:201-10. doi:10.1016/ S0360-3016(98)00544-6

6. Minohara S, Kanai T, Endo M, Noda K, Kanazawa M. Respiratory gated irradiation system for heavy-ion radiotherapy. Radiat Oncol Biol (2000) 47:1097-103. doi:10.1016/S0360-3016(00)00524-1 
7. Mizota M, Kanai T, Yusa K, Akagi T, Shimbo M, Yamashita H, et al. Reconstruction of biologically equivalent dose distribution on CT-image from measured physical dose distribution of therapeutic beam in water phantom. Phys Med Biol (2002) 47:935-45. doi:10.1088/0031-9155/47/6/306

8. Tsujii H, Kamada T, Shirai T, Noda K, Tsuji H, Karasawa K. In: Tsujii H, Kamada T, Shirai T, Noda K, Tsuji H, Karasawa K, editors. CarbonIon Radiotherapy. Tokyo: Springer Science \& Business Media (2013). p. 127-309.

9. Jermann M. Particle therapy statistics in 2014. Int J Part Ther (2015) 2:50-4. doi:10.14338/IJPT-15-00013

10. Combs SE, Debus J. Treatment with heavy charged particles: systematic review of clinical data and current clinical (comparative) trials. Acta Oncol (2013) 52:1272-86. doi:10.3109/0284186X.2013.818254

11. Blattmann C, Oertel S, Schulz-Ertner D, Rieken S, Haufe S, Ewerbeck V, et al. Non-randomized therapy trial to determine the safety and efficacy of heavy ion radiotherapy in patients with non-resectable osteosarcoma. BMC Cancer (2010) 10:96. doi:10.1186/1471-2407-10-96

12. Combs SE, Kessel KA, Herfarth K, Jensen A, Oertel S, Blattmann C, et al. Treatment of pediatric patients and young adults with particle therapy at the Heidelberg Ion Therapy Center (HIT): establishment of workflow and initial clinical data. Radiat Oncol (2012) 7:170. doi:10.1186/1748-717X-7-170

13. Combs SE, Kieser M, Habermehl D, Weitz J, Jäger D, Fossati P, et al. Phase I/II trial evaluating carbon ion radiotherapy for the treatment of recurrent rectal cancer: the PANDORA-01 trial. BMC Cancer (2012) 12:137. doi:10.1186/1471-2407-12-137

14. Combs SE, Habermehl D, Kieser M, Dreher C, Werner J, Haselmann R, et al. Phase I study evaluating the treatment of patients with locally advanced pancreatic cancer with carbon ion radiotherapy: the PHOENIX-01 trial. BMC Cancer (2013) 13:419. doi:10.1186/1471-2407-13-419

15. Brown A, Suit H. The centenary of the discovery of the Bragg peak. Raiother Oncol (2004) 73(3):265-8. doi:10.1016/j.radonc.2004.09.008

16. Durante M, Loeffler JS. Charged particles in radiation oncology. Nature Reviews Clinical Oncology (2010) 7:37-43. doi:10.1038/nrclinonc.2009.183

17. Raju MR, Carpenter SG. A heavy particle comparative study. Part IV: acute and late reactions. Br J Radiol (1978) 51:720-7. doi:10.1259/0007-1285-51-609-720

18. Tsujii H, Kamada T. A review of update clinical results of carbon ion radiotherapy. Jpn J Clin Oncol (2012) 42:670-85. doi:10.1093/jjco/hys104

19. Kamada T, Tsujii H, Blakely EA, Debus J, De Neve W, Durante M, et al. Carbon ion radiotherapy in Japan: an assessment of 20 years of clinical experience. Lancet Oncol (2015) 16:e93-100. doi:10.1016/S1470-2045(14)70412-7

20. Imada $H$, Kato $H$, Yasuda $S$, Yamada $S$, Yanagi $T$, Kishimoto $R$, et al. Comparison of efficacy and toxicity of short-course carbon ion radiotherapy for hepatocellular carcinoma depending on their proximity to the porta hepatis. Radiother Oncol (2010) 96:231-5. doi:10.1016/j.radonc.2010.05.019

21. Shinoto M, Yamada S, Terashima K, Yasuda S. Carbon-ion radiotherapy with concurrent gemcitabine for patients with locally advanced pancreatic cancer. Int J Radiat Oncol (2015) 95:498-504. doi:10.1016/j.ijrobp.2015.12.362

22. Okada T, Tsuji H, Kamada T, Akakura K, Suzuki H, Shimazaki J, et al. Carbon ion radiotherapy in advanced hypofractionated regimens for prostate cancer: from 20 to 16 fractions. Int J Radiat Oncol Biol Phys (2012) 84:968-72. doi:10.1016/j.ijrobp.2012.01.072

23. Nomiya T, Tsuji H, Maruyama K, Toyama S, Suzuki H, Akakura K, et al. Phase I/II trial of definitive carbon ion radiotherapy for prostate cancer: evaluation of shortening of treatment period to 3 weeks. Br J Cancer (2014) 110:2389-95. doi:10.1038/bjc.2014.191

24. Ishikawa H, Tsuji H, Kamada T, Akakura K, Suzuki H, Shimazaki J, et al. Carbon-ion radiation therapy for prostate cancer. Int J Urol (2012) 19:296-305. doi:10.1111/j.1442-2042.2012.02961.x

25. Mizoe J-E, Hasegawa A, Jingu K, Takagi R, Bessyo H, Morikawa T, et al. Results of carbon ion radiotherapy for head and neck cancer. Radiother Oncol (2012) 103:32-7. doi:10.1016/j.radonc.2011.12.013

26. Jingu K, Tsujii H, Mizoe J-E, Hasegawa A, Bessho H, Takagi R, et al. Carbon ion radiation therapy improves the prognosis of unresectable adult bone and soft-tissue sarcoma of the head and neck. Int J Radiat Oncol Biol Phys (2012) 82:2125-31. doi:10.1016/j.ijrobp.2010.08.043

27. Hasegawa A, Koto M, Takagi R. Carbon-ion radiotherapy for malignant head and neck cancer. NIRS \& MedAustron Joint Symposium on Carbon Ion
Radiotherapy, 2013. (2014). p. 6-13. Available from: http://www.nirs.go.jp/ publication/proceedings/NIRS_MedAustron/proceedings.pdf

28. Schulz-Ertner D, Nikoghosyan A, Didinger B, Münter M, Jäkel O, Karger CP, et al. Therapy strategies for locally advanced adenoid cystic carcinomas using modern radiation therapy techniques. Cancer (2005) 104:338-44. doi:10.1002/ cncr.21158

29. Kamada T, Tsujii H, Tsuji H, Yanagi T, Mizoe J, Miyamoto T, et al. Efficacy and safety of carbon ion radiotherapy in bone and soft tissue sarcomas. J Clin Oncol (2002) 20:4466-71. doi:10.1200/JCO.2002.10.050

30. Imai R, Kamada T, Sugahara S, Tsuji H, Tsujii H. Carbon ion radiotherapy for sacral chordoma. Br J Radiol (2011) 84:S48-53. doi:10.1259/bjr/13783281

31. Matsunobu A, Imai R, Kamada T, Imaizumi T, Tsuji H, Tsujii H, et al. Impact of carbon ion radiotherapy for unresectable osteosarcoma of the trunk. Cancer (2012) 118:4555-63. doi:10.1002/cncr.27451

32. Matsumoto K, Imai R, Kamada T, Maruyama K, Tsuji H, Tsujii H, et al. Impact of carbon ion radiotherapy for primary spinal sarcoma. Cancer (2013) 119:3496-503. doi:10.1002/cncr.28177

33. Serizawa I, Kagei K, Kamada T, Imai R, Sugahara S, Okada T, et al. Carbon ion radiotherapy for unresectable retroperitoneal sarcomas. Radiat Oncol Biol (2009) 75:1105-10. doi:10.1016/j.ijrobp.2008.12.019

34. Mizoe J-E, Hasegawa A, Takagi R, Bessho H, Onda T, Tsujii H. Carbon ion radiotherapy for skull base chordoma. Skull Base (2009) 19:219-24. doi:10.1 055/s-0028-1114295

35. Koto M, Hasegawa A, Takagi R, Fujikawa A, Morikawa T, Kishimoto R, et al. Risk factors for brain injury after carbon ion radiotherapy for skull base tumors. Radiother Oncol (2014) 111:25-9. doi:10.1016/j.radonc.2013. 11.005

36. Schulz-Ertner D, Karger CP, Feuerhake A, Nikoghosyan A, Combs SE, Jäkel $\mathrm{O}$, et al. Effectiveness of carbon ion radiotherapy in the treatment of skull-base chordomas. Radiat Oncol Biol (2007) 68:449-57. doi:10.1016/j. ijrobp.2006.12.059

37. Schulz-Ertner D, Nikoghosyan A, Hof H, Didinger B, Combs SE, Jäkel O, et al. Carbon ion radiotherapy of skull base chondrosarcomas. Radiat Oncol Biol (2007) 67:171-7. doi:10.1016/j.ijrobp.2006.08.027

38. Miyamoto T, Baba M, Sugane T, Nakajima M, Yashiro T, Kagei K, et al. Carbon ion radiotherapy for stage I non-small cell lung cancer using a regimen of four fractions during 1 week. J Thorac Oncol (2007) 2:916-26. doi:10.1097/ JTO.0b013e3181560a68

39. Yamamoto N. Carbon ion radiotherapy for lung cancer. HIMAC International Symposium. (2015). Available from: http://www.nirs.go.jp/ENG/publication/ proceedings/pdf/HIMAC_International_Symposium2015.pdf

40. Yasuda S, Isozaki Y, Yamada S. Carbon ion radiotherapy for hepatocellular carcinoma. HIMAC International Symposium. (2015). Available from: http:// www.nirs.go.jp/ENG/publication/proceedings/pdf/HIMAC_International_ Symposium2015.pdf

41. Hennequin C, Quero L, Rivera S. Radiosensitivity of hepatocellular carcinoma. Cancer Radiother (2011) 15:39-42. doi:10.1016/j.canrad.2010.11.004

42. Yasuda S, Harada M, Yamada S, Shiomi M, Isozaki T, Kamada T. Carbon-ion radiotherapy for hepatocellular carcinoma. Conference Proceedings of NIRSMedAustron Joint Conference on Carbon-Ion Radiotherapy. (2013).

43. Habermehl D, Debus J, Ganten T, Ganten M-K, Bauer J, Brecht IC, et al. Hypofractionated carbon ion therapy delivered with scanned ion beams for patients with hepatocellular carcinoma - feasibility and clinical response. Radiat Oncol (2013) 8:59. doi:10.1186/1748-717X-8-59

44. Yamada S, Anzai M, Okada N. Carbon ion radiotherapy for patients with pelvic recurrence of rectal cancer. HIMAC International Symposium. (2015). Available from: http://www.nirs.go.jp/ENG/publication/proceedings/pdf/ HIMAC_International_Symposium2015.pdf

45. Yamada S, Kamada T, Ebner DK, Shinoto M, Maier A, Terashima K, et al. Carbon-ion radiotherapy for pelvic recurrence of rectal cancer. Int J Radiat Oncol (2016). doi:10.1016/j.ijrobp.2016.04.022

46. Wakatsuki M, Kato S, Ohno T, Karasawa K, Kiyohara H, Tamaki T, et al. Clinical outcomes of carbon ion radiotherapy for locally advanced adenocarcinoma of the uterine cervix in phase $1 / 2$ clinical trial (protocol 9704). Cancer (2014) 120:1663-9. doi:10.1002/cncr.28621

47. Combs SE, Bruckner T, Mizoe J-E, Kamada T, Tsujii H, Kieser M, et al. Comparison of carbon ion radiotherapy to photon radiation alone or in 
combination with temozolomide in patients with high-grade gliomas: explorative hypothesis-generating retrospective analysis. Radiother Oncol (2013) 108:132-5. doi:10.1016/j.radonc.2013.06.026

48. Mizoe J-E, Tsujii H, Hasegawa A, Yanagi T, Takagi R, Kamada T, et al. Phase I/II clinical trial of carbon ion radiotherapy for malignant gliomas: combined $\mathrm{X}$-ray radiotherapy, chemotherapy, and carbon ion radiotherapy. Radiat Oncol Biol (2007) 69:390-6. doi:10.1016/j.ijrobp.2007.03.003

49. Hasegawa A, Mizoe J-E, Tsujii H, Kamada T, Jingu K, Iwadate Y, et al. Experience with carbon ion radiotherapy for WHO Grade 2 diffuse astrocytomas. Int J Radiat Oncol Biol Phys (2012) 83:100-6. doi:10.1016/j. ijrobp.2011.06.1952

50. Mori S, Zenklusen S, Inaniwa T, Furukawa T, Imada $\mathrm{H}$, Shirai $\mathrm{T}$, et al. Conformity and robustness of gated rescanned carbon ion pencil beam scanning of liver tumors at NIRS. Radiother Oncol (2014) 111:431-6. doi:10.1016/j. radonc.2014.03.009

51. Mori S. Real-time image-processing algorithm for markerless tumour tracking using X-ray fluoroscopic imaging. Br J Radiol (2014) 87:20140001. doi:10.1259/bjr.20140001

52. Shirai T. Hardware development and medical physics. 2nd International Symposium on Heavy-Ion Radiotherapy and Advanced Technology; 2016 Jan 9; Tokyo (2016). p. 10-14. Available from: http://www.nirs.qst.go.jp/rd/reports/ proceedings/pdf/2nd_International_Symposium_2016.pdf

53. Mobaraki A, Ohno T, Yamada S, Sakurai H, Nakano T. Cost-effectiveness of carbon ion radiation therapy for locally recurrent rectal cancer. Cancer Sci (2010) 101:1834-9. doi:10.1111/j.1349-7006.2010.01604.x

54. Ohno T. Particle radiotherapy with carbon ion beams. EPMA J (2013) 4:9. doi:10.1186/1878-5085-4-9
55. Jäkel O, Land B, Combs SE, Schulz-Ertner D, Debus J. On the cost-effectiveness of carbon ion radiation therapy for skull base chordoma. Radiother Oncol (2007) 83:133-8. doi:10.1016/j.radonc.2007.03.010

56. Tommasino F, Scifoni E, Durante M. New ions for therapy. Int J Part Ther (2015) 2:428-38. doi:10.14338/IJPT-15-00027.1

57. Inaniwa $\mathrm{T}$, Kanematsu N, Matsufuji N, Kanai T, Shirai T, Noda K, et al Reformulation of a clinical-dose system for carbon-ion radiotherapy treatment planning at the National Institute of Radiological Sciences, Japan. Phys Med Biol (2015) 60:3271-86. doi:10.1088/0031-9155/60/8/3271

58. Fossati P, Molinelli S, Matsufuji N, Ciocca M, Mirandola A, Mairani A, et al. Dose prescription in carbon ion radiotherapy: a planning study to compare NIRS and LEM approaches with a clinically-oriented strategy. Phys Med Biol (2012) 57:7543-54. doi:10.1088/0031-9155/57/22/7543

59. Tinganelli W, Durante M, Hirayama R, Krämer M, Maier A, KraftWeyrather W, et al. Kill-painting of hypoxic tumours in charged particle therapy. Sci Rep (2015) 5:17016. doi:10.1038/srep17016

Conflict of Interest Statement: The authors declare that the research was conducted in the absence of any commercial or financial relationships that could be construed as a potential conflict of interest.

Copyright $\odot 2016$ Ebner and Kamada. This is an open-access article distributed under the terms of the Creative Commons Attribution License (CC BY). The use, distribution or reproduction in other forums is permitted, provided the original author(s) or licensor are credited and that the original publication in this journal is cited, in accordance with accepted academic practice. No use, distribution or reproduction is permitted which does not comply with these terms. 